

\title{
Astrophysical and Structural Parameters of the Open Clusters NGC 6866, NGC 7062, and NGC 2360
}

\author{
Orhan Güneş ş,1,*, Yüksel Karataş $^{\mathrm{b}, 1}$, Charles Bonatto ${ }^{\mathrm{c}, 1}$ \\ ${ }^{a}$ Department of Astronomy and Space Sciences, Faculty of Arts and Sciences, Erciyes \\ University, Talas Yolu, 38039, Kayseri, Turkey \\ ${ }^{b}$ Istanbul University, Science Faculty, Department of Astronomy and Space Sciences, 34119 \\ University, Istanbul, Turkey \\ ${ }^{c}$ Universidade Federal do Rio Grande do Sul, Departamento de Astronomia, CP 15051, RS, \\ Porto Alegre 91501-970, Brazil
}

\begin{abstract}
We derive astrophysical and structural parameters of the poorly studied open clusters NGC 6866, NGC 7062, and NGC 2360 based on filtered 2MASS ( $J, J-$ $H$ ) diagrams, and stellar radial density profiles. The field star decontamination technique is utilised for selecting high-probability cluster members. The $E(B-$ $V$ ) reddening values of the three clusters derived from $2 \mathrm{MASS} J K_{s}$ agree with those inferred from UBV and $u v b y-\beta$ photometries. We find that the core mass function slopes are flatter than the halo's for the three clusters. The large core and cluster radii of NGC 6866 and NGC 2360 indicate an expanded core, which may suggest the presence of stellar mass black-holes. NGC 2360 is located in the third quadrant $\left(\ell=229^{\circ} .80\right)$, where Giant Molecular Clouds are scarce that, together with its relatively large mass $\left(\sim 1800 \mathrm{~m}_{\odot}\right)$, might explain its longevity $(\sim 1.8 \mathrm{Gyr})$ in the Galaxy.
\end{abstract}

Keywords: (Galaxy:) open clusters and associations:general, Galaxy: open clusters and associations:individual, Galaxy: stellar content

PACS 97.10 Wn; 97.80.Fk; 97.80.Hn

\section{Introduction}

Reliable astrophysical and structural parameters of Galactic open clusters (OCs) are an excellent source of information for interpreting their dynamics, and the 2MASS JHK $K_{s}$ photometric data base provides a unique opportunity to derive these kind of parameters in a uniform way.

The stellar content of OCs undergoes internal and external changes related to, e.g. stellar evolution, mass segregation, and encounters with the disk and Giant Molecular Clouds (GMCs) (Lamers \& Gieles, 2006), (Gieles et al., 2007).

\footnotetext{
* Corresponding author

${ }^{1}$ E-mail: or.gunes@gmail.com
} 
Combined, these process produce a varying degree of mass loss that may lead to the cluster dissolution into the field. For instance, the relative lack of old OCs in the solar vicinity can be partly explained by encounters with GMCs. The mass function slope $\chi$, relaxation time and evolutionary parameters are required (Bonatto \& Bica , 2005, 2007a; Bica et al., 2006a) in order to be able to see mass segregation effects in the stellar content of OCs.

In this paper we focus on the above issues on three poorly studied OCs based on the improved field star decontamination procedure via 2MASS $\mathrm{JH}_{s}$ photometry (Bonatto \& Bica, 2007a b) and (Camargo et al., 2010).

This paper is organised as follows. In Section 2 the 2MASS photometry and the field star decontamination algorithm (employed in the CMD analyses) are presented. The derivation of astrophysical and structural parameters, and a comparison with the literature, are given in Sections 3 to 5 . Section 6 is dedicated to mass function properties of the clusters. Discussion of the results are in Section 7. Finally, in Section 8 a conclusion is given.

\section{2MASS JHK $K_{s}$ photometry and Field-star decontamination}

The $\mathrm{JH}_{s}$ photometry of 2MASS2 catalogue Skrutskie et al. (2006) has been used to find the cluster members of the OCs NGC 6866, NGC 7062, and NGC 2360. For this, VizieR ${ }^{3}$ was used to extract J, H, and $K_{s}$ 2MASS photometry for a large-area centred on these clusters. The XDSS4 $\mathrm{R}$ images of NGC 6866, NGC 7062, NGC 2360 are displayed in Fig. 1.

The technique used here for determining the cluster members of the three clusters is the field star decontamination procedure coupled to the 2MASS $\mathrm{JH} K_{s}$ photometry. It samples photometric properties of the stars in a comparison field to (statistically) remove the field contamination from the cluster CMD. This technique has been succesfully used by Bonatto \& Bica (2007a b $)$, Bonatto \& Bica (2008), Bica et al. (2008a), and Camargo et al. (2009), and applied to NGC 6866, NGC 7062, and NGC 2360. First, the stellar surface density $\sigma\left(\right.$ stars $\left.\operatorname{arcmin}^{-2}\right)$ of the raw data of these three clusters, computed for a mesh size of $3^{\prime} \times 3^{\prime}$ and centred on the coordinates in Table 1, has been displayed in the top panels of Figs. 2-4. The isopleth surfaces of these clusters are presented in the bottom panels of Figs. 2-4. From the stellar radial density profile (hereafter RDP) of each cluster, built based on the $\mathrm{JH} K_{s}$ photometry extracted for the WEBDA5 coordinates in Table 1, the cluster radii are taken to be $R=19^{\prime} .07$ (NGC 6866), $R=7^{\prime} .53$ (NGC 7062) and $R=22^{\prime} .63$ (NGC 2360), respectively (see Col. 10 of Table 4 ). The stellar RDP is the projected number of stars per area around the cluster centre. To avoid oversampling near the centre and undersampling for large radii, the RDPs are built by counting stars

\footnotetext{
${ }^{2}$ The Two Micron All Sky Survey, available at http://www.ipac.caltech.edu/2mass/releases/allsky/

${ }^{3}$ http://vizier.u-strasbg.fr/viz-bin/VizieR?-source=II/246.

${ }^{4}$ Extracted from the Canadian Astronomy Data Centre (CADC), at http://www.cadcwww.dao.nrc.ca/

${ }^{5}$ obswww.univie.ac.at/WEBDA-Mermilliod \& Paunzen (2003)
} 
in concentric rings of increasing width with distance to the centre. The number and width of rings are optimised so that the resulting RDPs have adequate spatial resolution with moderate $1 \sigma$ Poisson errors. The residual background level of each RDP corresponds to the average number of CM-filtered stars measured in the comparison field.

Consequently, stars within these cluster radii are considered to be probable cluster members. As the stellar comparison field, a wide external ring $\left(R=15^{\prime}-20^{\prime}\right)$ has been considered to eliminate field stars. As Camargo et al. (2010) noted, RDPs of OCs built based on the WEBDA coordinates usually show a dip in the inner RDP region. For this reason, new central coordinates of these clusters have been searched to maximise the star counts at the innermost RDP bin. Then, the 2MASS photometry was extracted again, but now centered on the optimised cluster coordinates. The optimised central coordinates are displayed in the panels of Fig. 1 as small circles, and given in the right section of Table 1. To have the intrinsic CMD morphology of the clusters, the statistical field star decontamination procedure of Bonatto \& Bica (2007a) is used. This procedure is based on the relative number densities of stars in a cluster region and offset field. It divides the full range of magnitudes and colours of a CMD into the cell dimensions of $\Delta J=1.0$, and $\Delta(J-H)=\Delta\left(J-K_{s}\right)=0.15$. These dimensions are adequate to allow sufficient star counts in individual cells and preserve the intrinsic morphology of the evolutionary sequences. As shown in Bonatto \& Bica (2007a), the field star decontamination procedure with 2MASS $\mathrm{JH} K_{s}$ is efficient in isolating stars with a high probability of being cluster members. Details on the algorithm can be found in Bonatto \& Bica (2007a,b) and Bonatto \& Bica (2009a,b,c), and Camargo et al. (2010).

\section{Astrophysical parameters}

Having applied the field star decontamination technique to eliminate field stars, the decontaminated $(J, J-H)$ CMDs of NGC 6866, NGC 7062, and NGC 2360 are plotted in Figs. 5(a)-(c). The shaded area in the figures are the colour-magnitude filters, which follow the distribution of the decontaminated star sequences in the CMDs. These filters are wide enough to accommodate the colour distributions of main sequence and evolved stars of the clusters, allowing $1 \sigma$ photometric uncertainties. The solid lines in Figs. 5(a)-(c) represent the fitted 0.8, 1 and 1.8 Gyr Padova isochrones (Girardi et al. 2002, hereafter G02) for $Z=+0.019$ (solar) abundance. As can be seen from Figs. 5(a) $-(\mathrm{c})$, the G02 isochrones fit well the main sequence (MS), turn-off (TO) and RC/RG regions on the CMDs of the clusters. Due to the presence of binaries, the G02 isochrones have been shifted to the left of the main sequence in Figs. 5a-c (Bonatto \& Bica, 2008, see the Sect. 5).

The astrophysical parameters (reddening, distance modulus, distance and age) of three clusters have been derived based on the solar metallicity isochrones with the ages 0.8, 1 and 1.8 Gyr G02 isochrones fitted to the decontaminated CMDs. We find the reddenings $E(J-H)=0.06 \pm 0.02$ for NGC 6866, $E(J-H)$ $=0.10 \pm 0.02$ for NGC 7062, $E(J-H)=0.02 \pm 0.01$ for NGC 2360, respectively, 
from the CMD diagrams of Figs. 5(a) - (c). These reddenings in $E(J-H)$ are converted to $E(B-V)$ by considering the relations $A_{J} / A_{V}=0.276, A_{H} / A_{V}=$ $0.176, A_{K_{s}} / A_{V}=0.118, A_{J}=2.76 \times E(J-H)$, and $E(J-H)=0.33 \times$ $E(B-V)$ (Dutra et al., 2002), assuming a constant total-to-selective absorption ratio $R_{V}=3.1$. The resulting $E(B-V)$ values are given in Col. 3 of Table 2. Following the same procedure, the distance moduli of these clusters have been derived and listed in Col. 4 of Table 2. The estimated heliocentric $d(k p c)$ and Galactocentric $R_{G C}$ (kpc) distances are given in Cols. 5-6, respectively. When estimating the $R_{G C}$ distances, the value $R_{\odot}=7.2 \pm 0.3 \mathrm{kpc}$ of Bica et al. $(2006 \mathrm{~b})$ is considered.

The reddening values of NGC 6866, NGC 7062, and NGC 2360 have been compared to those of the dust maps of Schlegel et al. (1998, hereafter SFD). These are based on the COBE/DIRBE and IRAS/ISSA maps, and take into account the dust absorption all the way to infinity. SFD dust maps give $E(B-$ $V)_{\infty}$ as 0.72 for NGC 6866, 1.15 for NGC 7062, 0.80 for NGC 2360. The distances in Col. 5 of Table 2 is used for the reduced reddening. By following the method of Bonifacio et al. (2000), the reduced final reddenings are $E(B-V)_{A}=$ $0.42,0.42$, and 0.18 for NGC 6866, NGC 7062, and NGC 2360, respectively. Within the uncertainties the reddening value of $0.32 \pm 0.06$ of 2MASS $\mathrm{JH} K_{s}$ photometry for NGC 7062 is close to the value of 0.42 obtained from the dust maps of SFD. Our reddening values of 0.19 for NGC 6866 and 0.06 for NGC 2360 are lower than $E(B-V)=0.42$ and 0.18 of the dust maps of SFD, respectively.

\section{Comparison with the literature}

A comparison of the present astrophysical parameters with those in the literature is given in Table 3. Our (2MASS) value of $E(B-V)=0.19 \pm 0.06$ for NGC 6866 agrees with the $(0.17, U B V)$ of Hoag et al. (1961); the same applies to our $E(B-V)=0.06 \pm 0.03$ of NGC 2360 with respect to the $(0.07, U B V)$ of Eggen (1968). However, our reddening value of $E(B-V)=0.32 \pm 0.06$ of NGC 7062 is relatively far from the $(0.46, u b v y-\beta)$ of Peniche et al. (1990), which might be partly explained by the very different metallicities used in both studies.

As can seen in Cols. 5-6 of Table 3, within the uncertainties the literature distance moduli and distances of NGC 6866 and NGC 2360 are relatively close to the values of this paper. The age of NGC 6866 (from the 2MASS JHK $K_{s}$ photometry) of this paper is older than 0.38 Gyr value of Hoag et al. (1961). Although the reddenings and heavy element abundances between this paper and Hoag et al. (1961) are almost the same, this difference in ages results from the adopted isochrones. The older study of Hoag et al. (1961) considers the observational ZAMS. This paper considers the G02 isochrone, which contains the new input physics. Since Eggen (1968) did not give an age value for NGC 2360, no any comparison was done.

The distance modulus and distance of NGC 7062 are fainter and larger as compared to the result of Peniche et al. (1990). Our age value of $1.0 \mathrm{Gyr}$, derived from 2MASS $\mathrm{JH}_{s}$ photometry is quite older than the 0.28 Gyr given 
by Peniche et al. (1990). These differences stem from the metal and heavy element abundance approximations between this paper and Peniche et al. (1990). Peniche et al. (1990) measure the abundances of NGC 7062 as $([\mathrm{Fe} / \mathrm{H}], \mathrm{Z})=$ $(-0.35,+0.003)$ from $u b v y-\beta$ photometry, and consider the isochrone of VandenBerg \& Clem (1985) for $Z=+0.003$, whereas we adopt the solar metallicity G02 isochrones.

\section{Structural parameters}

Structural parameters of NGC 6866, NGC 7062, and NGC 2360 are derived by means of the stellar radial density profile (RDP), which is explained in Section 2. Usually, the RDPs of star clusters can be described by an analytical profile, like the empirical, single mass, modified isothermal spheres of King (1966) and Wilson (1975), and the power law with a core of Elson et al. (1987). These functions are characterised by different sets of parameters that are related to the cluster structure. We adopt the two-parameter function $\sigma(R)=\sigma_{b g}+\sigma_{0} /\left(1+\left(R / R_{c}\right)^{2}\right)$, where $\sigma_{b g}$ is the residual background density, $\sigma_{0}$ the central density of stars, and $R_{\text {core }}$ the core radius. Applied to star counts, this function is similar to that used by King (1962) to describe the surface brightness profiles in the central parts of globular clusters. The RDPs of the clusters NGC 6866, NGC 7062, and NGC 2360, fitted with King profiles are shown in Figs. 6(a)-(c). In Fig.6 the solid line shows the best fit King profile, horizontal red bar denotes the stellar background level measured in the comparison field, and the $1 \sigma$ King fit uncertainty is shown by the shaded domain. The cluster radius $\left(R_{R D P}\right)$ is also obtained from the measuring the distance from the cluster centre where the RDP and residual background are statistically indistinguishable (Bonatto \& Bica, 2007a). The $R_{R D P}$ can be taken as an observational truncation radius, whose value depends both on the radial distribution of member stars and the field density. These structural parameters and their meanings are listed in Table 4.

\section{Mass and Mass functions}

The stellar masses stored in NGC 6866, NGC 7062, and NGC 2360 have been determined by means of their mass functions (MFs), built for the observed MS mass range according to Bica et al. (2006a). By following the algorithm, which is basically defined by Bonatto \& Bica (2005), luminosity functions from the decontaminated $(J, J-H)$ diagrams of the three clusters have been transformed into MFs through the corresponding mass-luminosity relations derived from Padova isochrones for the ages in Col. 2 of Table 2. The relations of $\phi(m)\left(\right.$ stars $\left.m_{\odot}^{-1}\right)$ versus $m_{\odot}$ of NGC 6866 , NGC 7062, and NGC 2360 are shown in Figs. 7-9 for different cluster regions. The mass ranges shown in Figs. $7-9$ are $m=1.13-2.23 m_{\odot}$ for NGC $6866, m=1.08-1.93 m_{\odot}$ for NGC 7062 , and $m=0.83-1.63 m_{\odot}$ for NGC 2360 , respectively.

The MS MFs in Figs. $7-9$ are fitted with the function $\phi(m) \propto m^{-(1+\chi)}$, and the MF slopes $(\chi)$ have been determined for the different regions, which are 
indicated in Figs. 7-9 and Col. 1 of Table 5. Details of this approach are given in Table 5, where we also show the number and mass of the evolved stars. The lower MS is not accessed on the $(J, J-H)$ diagrams of the three clusters, but we assume that the low-mass content is still present, and use Kroupa's MF6 to estimate the total stellar mass, down to the H-burning mass limit $\left(0.08 m_{\odot}\right)$. The results (number of stars, MS and evolved star stellar contents, MF slope $(\chi)$, and mass extrapolated to $\left.0.08 m_{\odot}\right)$ for each cluster region are given in Table 5.

The MF slopes of NGC 6866 (Fig. 7) are quite flat $(\chi=-1.37 \pm 0.54)$ in the core and very steep $(\chi=+2.23 \pm 0.49)$ in the region of $r=[1.97,9.15] \mathrm{pc}$. Such a MF slope steeping from the core to the outskirts of a cluster implies that core low-mass stars are being transferred to the cluster's halo, while massive stars accumulate in the core, due to mass segregation. The overall MF slope $(\chi=+1.32 \pm 0.46)$ for $r=[0,9.15] \mathrm{pc}$ is quite close to the standard Salpeter IMF value (Salpeter, 1955) of $\chi=+1.35$.

The MF slope is also flat $(\chi=-0.66 \pm 0.60)$ in the core of NGC 7062 cluster, whereas MF slope is very steep with $\chi=+2.28 \pm 0.44$ in the region of $r=[0.66,3.59] \mathrm{pc}$. The overall MF slope is also very steep with $\chi=+2.16 \pm 0.33$ for $r=[0,3.59] \mathrm{pc}$, which is different from Salpeter's value. The numbers of low mass and high mass stars in the core of NGC 7062 are almost equivalent. As seen from Fig. 8, the variation in $\chi$ is quite large from the core to the halo of the cluster NGC 7062, due to the large scale mass segregation.

For the cluster NGC 2360, the MF slope is quite flat $(\chi=-0.14 \pm 0.60)$ in the core, increasing to $\chi=+1.07 \pm 0.48$ in the region $r=[1.13,6.85] \mathrm{pc}$. The overall MF slope is $\chi=+0.67 \pm 0.36$ for $r=[0,6.85] \mathrm{pc}$, which is different from Salpeter's value. Flat core and overall MF slopes of this cluster imply mild mass segregation effects.

\section{Discussion}

The relaxation time $t_{r l x}$ is the characteristic time-scale for a cluster to reach some level of energy equipartition (Binnev \& Merrifield, 1998) and, as discussed in Bonatto \& Bica (2005), Bonatto \& Bica (2006), Bonatto \& Bica (2007a), the evolutionary parameter $\left(\tau=A g e / t_{r l x}\right)$ appears to be a good indicator of dynamical state. Following Bonatto \& Bica (2006), we parameterize $t_{r l x}$ as $t_{r l x} \approx 0.04\left(\frac{N}{\operatorname{lnN}}\right)\left(\frac{R}{1 p c}\right)$, where $\mathrm{N}$ is the number of stars located inside the region of radius $\mathrm{R}$. The uncertainties in the evolutionary parameters $(\tau)$ of the three clusters have been estimated by propagating the errors in Age (Table 2), Radii (Table 4) and N (Table 5) into $t_{r l x}$ and $\tau$. Although the errors in cluster radii and age (Tables 2 and 4$)$ are at a similar $(\sim 10-20 \%)$ level for the three clusters, the uncertainties in $R_{\text {core }}$ and especially in the number of stars $\mathrm{N}$ are

\footnotetext{
${ }^{6} \chi=0.3 \pm 0.5$ (Kroupa, 2001) for $0.08<m_{\odot}<0.5, \chi=1.3 \pm 0.3$ for $0.5<m_{\odot}<1.0$, and $\chi=1.3 \pm 0.7$ for $1.0<m_{\odot}$.
} 
much larger, particularly for NGC 7062 and NGC 2360 . When propagated, the latter two errors produce a large uncertainty in $t_{r l x}$ (Table 6) and, consequently, a large uncertainty in the evolutionary parameter. In this sense, both $t_{r l x}$ and $\tau$ should be taken simply as an order of magnitude estimate.

As emphasized by Bonatto \& Bica (2006), the two parameters present a signature that low-mass stars originally in the core are transferred to the cluster's outskirts, while massive stars sink in the core, which is related to masssegregation. Bonatto \& Bica (2005, 2006) discuss that the significant flattening in core and overall MFs due to dynamical effects such as mass segregation is expected to occur for $\tau_{\text {core }} \geq 100$ and $\tau_{\text {overall }} \geq 7$, respectively.

For a typical $\sigma_{v} \approx 3 \mathrm{kms}^{-1}$ (Binney \& Merrifield, 1998), the relaxation times $\left[t_{r l x}\right.$ (overall), $t_{r l x}($ core $\left.)\right]$ and the evolutionary parameters $\left[\tau_{\text {overall }}, \tau_{\text {core }}\right]$ for NGC 6866, NGC 7062, and NGC 2360 clusters are given in Table 6.

The very steep core MF of NGC $6866(\chi=-1.37)$ is due to the relaxation time and the evolutionary parameter of $\left[t_{r l x}(\right.$ core $\left.), \tau_{\text {core }}\right]=\left[\begin{array}{ll}1.77 \\ \mathrm{Myr}\end{array}, 453\right]$. The very steep halo and rather steep overall MFs $(\chi=+2.23, \chi=+1.32)$ present signs of large-scale mass segregation in the core/halo region, due to the $\left[t_{r l x}(\right.$ overall $\left.), \tau_{\text {overall }}\right]=[210 \mathrm{Myr}, 3.80]$.

For NGC 7062, the flat slope $(\chi=-0.66)$ in the core is not coincident with the values of $\left[t_{r l x}(\right.$ core $\left.), \tau_{\text {core }}\right]=[0.61 \mathrm{Myr}, 1640] . t_{\text {rlx }}($ core $)$ of this cluster is lower than the value of 7 , given by Bonatto \& Bica (2006) and Bica et al. (2006a), and quite short to occur a flat core because of mass segregation. A very flat core would have been expected for this cluster, due to large value of $\tau_{\text {core }}$. The very steep halo and overall MFs $(\chi=+2.28, \chi=+2.16)$ are in reasonable concordance with the relaxation times $\left[t_{r l x}\right.$ (overall), $\left.\tau_{\text {overall }}\right]=[77.80 \mathrm{Myr}, 12.80]$. The evolutionary parameters for this cluster suggest an advanced dynamical state.

With the $\left[t_{r l x}(\right.$ core $\left.), \tau_{\text {core }}\right]=[3.17$ Myr, 569], NGC 2360 has a flat core MF $(\chi=-0.14)$. The rather steep halo and flat overall MFs $(\chi=+1.07, \chi=+0.67)$ of this cluster present signs of mild mass segregation in the core/halo region, as a result from the $\left[t_{r l x}\right.$ (overall $\left.), \tau_{\text {overall }}\right]=[153 \mathrm{Myr}, 11.80]$.

As seen from the Galactocentric distance values in Col. 6 of Table 2 and Fig. 10, since these clusters are located at the Solar circle, tidal disruption due to both the Galactic field and GMCs seems to be efficient.

The cluster and core radii $\left(R_{R D P}, R_{\text {core }}\right)$ of the three clusters (Cols. $5-6$ of Table 4), confirm that the larger clusters tend to have larger cores, as shown by Camargo et al. (2010, Fig.13(a)). The cluster and core radii $\left(R_{R D P}, R_{\text {core }}\right)=(3.59,0.66)$ pc of NGC 7062, which is quite small, imply that it may have shrunk in size with time. Thus, for this cluster, we see the dynamical effects of both mass segregation and core collapse. The overall mass $m=1560 m_{\odot}$ of NGC 7062 also indicates that it is a survivor, despite these dynamical processes and the location of $R_{G C}=7.30 \mathrm{kpc}$.

NGC 6866 and NGC 2360 have large cluster and core radii (Cols.5-6 of Table 4), a fact that may be partly associated with large-scale mass segregation and/or related to initial conditions. Alternatively, cluster heating due to stellar mass black-holes that are scattered towards the halo or ejected from the cluster 
may also explain part of the expansion (Mackey et al., 2007). Negative values of $\chi$ of the cores also show that the massive stars tend to be concentrated in the cores of the two clusters.

The relation between core and cluster radii, the dependence of the cluster radii on the Galactocentric distance, and the relation between the core and cluster radii with the age of the three clusters are presented in Figs.11(a)-(d), together with the data of Camargo et al. (2009). NGC 7062 and NGC 6866 are almost near the bifurcation age of $\approx 1$ Gyr, given by Camargo et al. (2009). NGC 2360 is slightly away from this bifurcation limit. The relation of the core and cluster radii with age is presented in Figs.11(c)-(d). This relation is related to the cluster survival/dissociation rates, as emphasized by Camargo et al. (2009). As is seen from panel (b), the three clusters are inside the standard IAU value $R_{G C}=8.5 \mathrm{kpc}$, and quite close to the $R_{\odot}=7.2 \pm 0.3 \mathrm{kpc}$ of Bica et al. (2006b).

NGC 7062 has small cluster and core radii, $\left(R_{R D P}, R_{\text {core }}\right)=(3.59,0.66)$ pc, and is located in the shaded region, plotted from the data (open circles) of Camargo et al. (2009) (panel a). The flat core MF slope $(\chi=-0.66 \pm 0.60)$ and very steep overall MF slope $(\chi=+2.28 \pm 0.44)$ of this cluster show large scale mass segregation. NGC 7062 seems to be at stage of core shrinkage because of dynamical relaxation. A nebulae near this cluster can cause the small core and cluster sizes, as a result of destruction process. Alternatively, the small core and cluster sizes may be primordial, which may be related to the high molecular gas density in Galactic center directions (van den Berg et al. , 1991; Camargo et al., 2010).

NGC 2360 is located in the third quadrant $\left(\ell=229^{\circ} .80\right)$, where GMCs are scarce. Its longevity is probably related to the relatively large mass, $1800 m_{\odot}$, large cluster radii and location in the Galaxy. Also, flat core and overall MF slopes of NGC 2360 imply mild mass segregation effects.

NGC 6866 is located at $\ell=79^{\circ} .56$, as is seen in Table 1 . Due to the presence of GMCs, tidal effects from disk and Bulge crossings as external processes in this direction, this cluster is a survivor with the values of $1800 m_{\odot}$ and $R_{R D P}=9.15$ pc. Moreover, the very steep core slope $\chi=-1.37$ and quite steep overall slope $\chi=+1.32$ of NGC 6866 indicate that the mass segregation effects are quite efficient.

\section{Conclusions}

Our main conclusions are summarized as follows:

1. The astrophysical and structural parameteres of the poorly studied clusters, NGC 6866, NGC 7062, and NGC 2360 have been derived from the filtered 2MASS $(J, J-H)$ CMDs, and the stellar RDPs. The field star decontamination technique is utilised for separating the cluster members.

2. The astrophysical parameters (Age, E(B-V), d) are (0.80 Gyr, 0.19, 1.65 kpc) for NGC 6866, (1.0 Gyr, 0.32, 1.64 kpc) for NGC 7062, (1.80 Gyr, 0.06, 1.04 
kpc) for NGC 2360, respectively. These clusters are inside the Solar circle. The reduced final reddenings from the dust maps of SFD are $E(B-$ $V)_{A}=0.42,0.42$, and 0.18 for NGC 6866, NGC 7062, NGC 2360, respectively. The reddening value of $0.32 \pm 0.06$ of $2 \mathrm{MASS} \mathrm{JH} K_{s}$ photometry for NGC 7062 is close to the value of 0.42 obtained from the dust maps of SFD. Our reddening values of $E(B-V)=0.19$ and 0.06 for NGC 6866 and NGC 2360, respectively are lower than $E(B-V)=0.42$ and 0.18 of the dust maps of SFD. However, the SFD values resulted from line-of-sight integral throughout the Milky Way and with low spatial resolution, it is quite a normal thing to have different reddening values for these relatively close $(\sim 1 \mathrm{kpc})$ star clusters.

3. The reddening values, 0.19 and 0.06 for NGC 6866 and NGC 2360 clusters, derived by 2 MASS JH $K_{s}$ photometry are in good agreement with the values of $(0.17, U B V)$ of Hoag et al. (1961) and (0.07, $U B V)$ of Eggen (1968). However, our reddening value of $E(B-V)=0.32 \pm 0.06$ of NGC 7062 is relatively far from the $(0.46, u b v y-\beta)$ of Peniche et al. (1990), which might be partly explained by the very different metallicities used in both studies. Within the uncertainties, distance moduli and distances of NGC 6866 and NGC 2360 agree with the values of this paper. The distance modulus and distance of NGC 7062 are lower than the one of Peniche et al. (1990). The 0.80 Gyr age value of NGC 6866, derived from 2MASS JH $K_{s}$ photometry, is older than $0.38 \mathrm{Gyr}$ value of Hoag et al. (1961). Although the reddenings and heavy element abundances between this paper and Hoag et al. (1961) are almost the same, this difference in ages are due to the usage of distinct isochrones which corresponds to differing internal physics of the isochrones and photometric systems. The 1.0 Gyr age value of NGC 7062, derived from 2MASS JHK $K_{s}$ photometry, is quite older than the $0.28 \mathrm{Gyr}$ given by Peniche et al. (1990). These differences stem from the metal and heavy element abundance assumptions between this paper and Peniche et al. (1990).

4. NGC 6866 has MF slopes that are quite flat $(\chi=-1.37)$ in the core and very steep $(\chi=+1.32)$ in the region of $r=[0,9.15] \mathrm{pc}$. This suggests that low-mass stars in the core are being transferred to the cluster's outskirts, while massive stars accumulate in the core. NGC 6866 is in the direction of the Galactic centre, and due to the presence of GMCs, and the dissolution effects associated with tidal effects from disk and Bulge crossings, this cluster is a survivor with the values of $1800 m_{\odot}$ and $R_{R D P}=9.15$ pc. Moreover, this cluster has large core and cluster radii, and shows an expanded core possibly due to the presence of a stellar mass black-hole.

5. The flat core MF slope $(\chi=-0.66)$ and the steep overall MF slope $(\chi=+2.16)$ indicate that the variation in $\chi$ is quite large from the core to the outskirts of the cluster NGC 7062, due to the large scale mass segregation. With small cluster and core radii, $\left(R_{R D P}, R_{\text {core }}\right)=(3.59,0.66) \mathrm{pc}$, this cluster seems to be at stage of core shrinkage because of dynamical relaxation. A nebulae near this cluster may be responsible for the small core and cluster sizes, as a result of destruction process. The small core 
and cluster sizes may be primordial, probably related to the high molecular gas density in Galactic center directions (van den Berg et al. , 1991; Camargo et al., 2010).

6. The large core and cluster radii of NGC 2360 indicate an expanded core, which may suggest the presence of a stellar mass black-hole. NGC 2360 is located in the third quadrant $\left(\ell=229^{\circ} .80\right)$, where GMCs are rare that, together with the large mass and cluster radius, might explain its longevity $(\sim 1.8 \mathrm{Gyr})$. Also, flat core $(\chi=-0.14)$ and overall MF $(\chi=+1.07)$ slopes of this cluster imply mild mass segregation effects. 


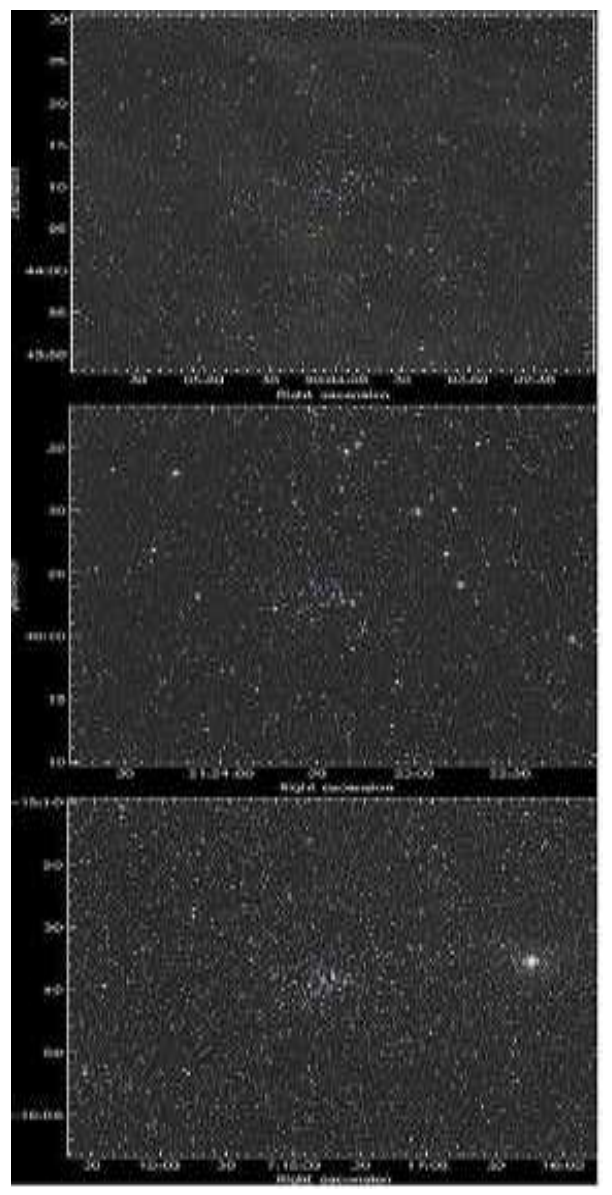

Figure 1: The images of NGC 6866 DSS-R $30^{\prime} \times 30^{\prime}$, NGC 7062 DSS-R $20^{\prime} \times 20^{\prime}$ and NGC 2360 DSS-R $40^{\prime} \times 40^{\prime}$, from top to bottom. 

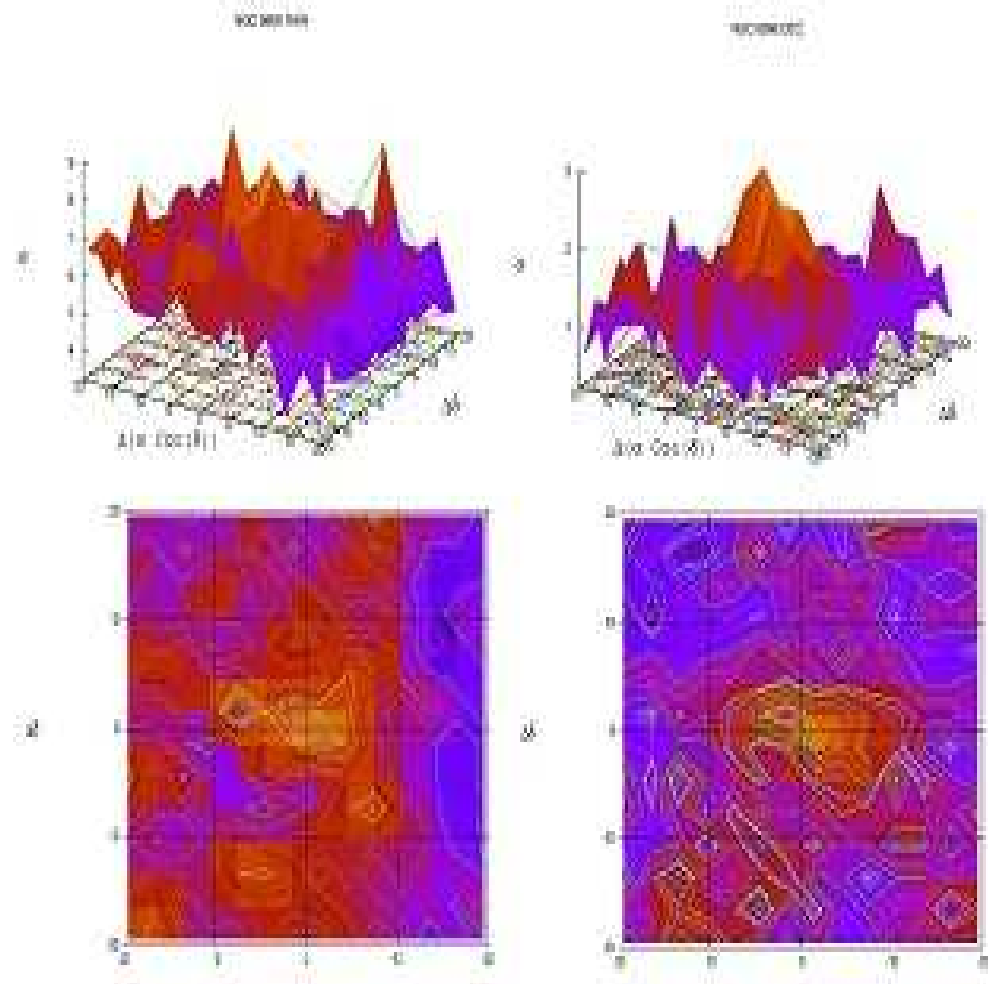

Hith

Whithit

Figure 2: For observed (raw) photometry, top panels: stellar surface-density $\sigma\left(\right.$ stars $\left.\operatorname{arcmin}^{-2}\right)$ of NGC 6866, computed for a mesh size of $3^{\prime} \times 3^{\prime}$, centred on the coordinates in Table 1. Bottom panels : The corresponding isopleth surfaces. Left panel: observed (raw) photometry. Right panel: decontaminated photometry. 

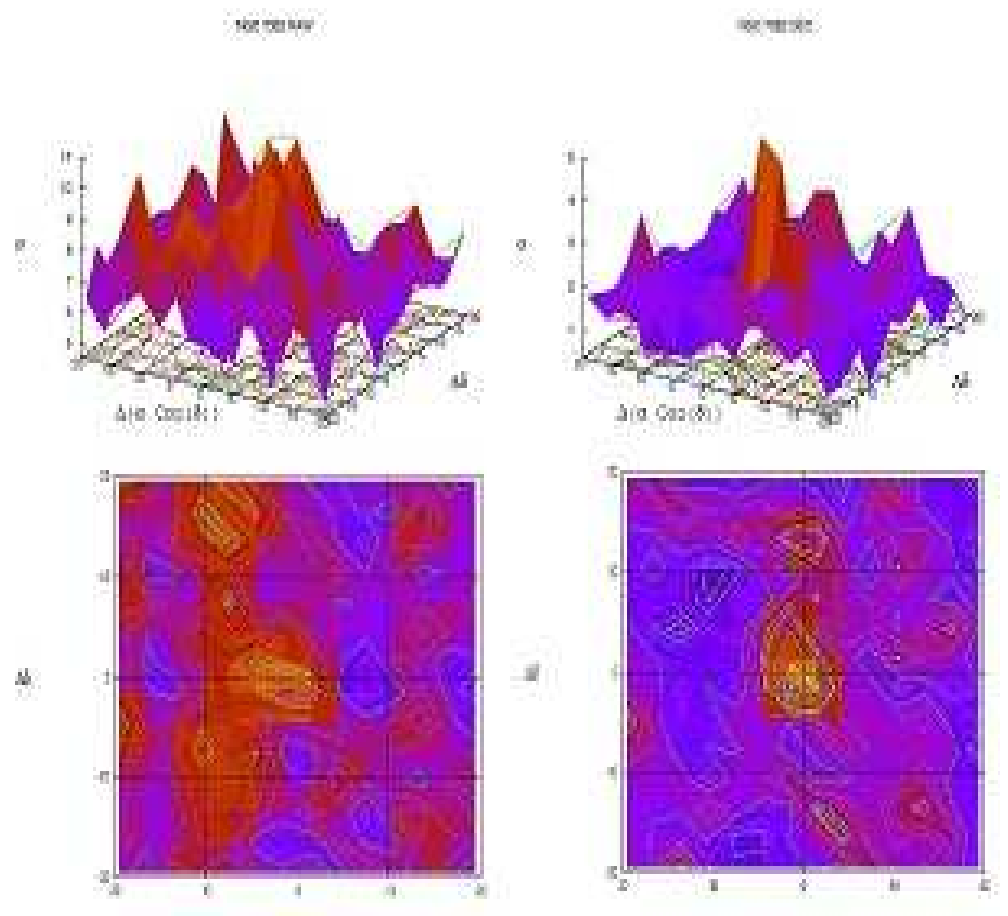

Hitaif

sin wh

Figure 3: For observed (raw) photometry, top panels: stellar surface-density $\sigma\left(\right.$ stars $\left.\operatorname{arcmin}^{-2}\right)$ of NGC 7062, computed for a mesh size of $3^{\prime} \times 3^{\prime}$, centred on the coordinates in Table 1. Bottom panels: The corresponding isopleth surfaces. Left panel: observed (raw) photometry. Right panel: decontaminated photometry. 

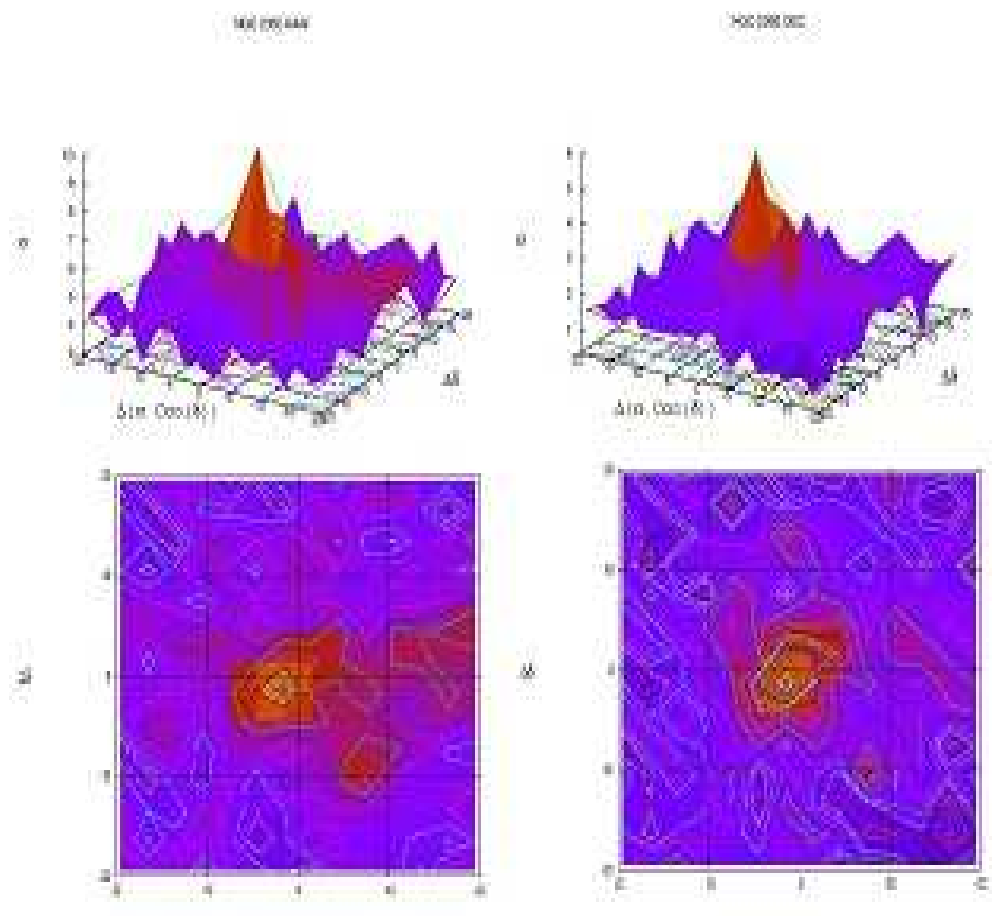

iil sophil

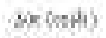

Figure 4: For observed (raw) photometry, top panels: stellar surface-density $\sigma\left(\right.$ stars $\left.\operatorname{arcmin}^{-2}\right)$ of NGC 2360, computed for a mesh size of $3^{\prime} \times 3^{\prime}$, centred on the coordinates in Table 1. Bottom panels : The corresponding isopleth surfaces. Left panel: observed (raw) photometry. Right panel: decontaminated photometry. 

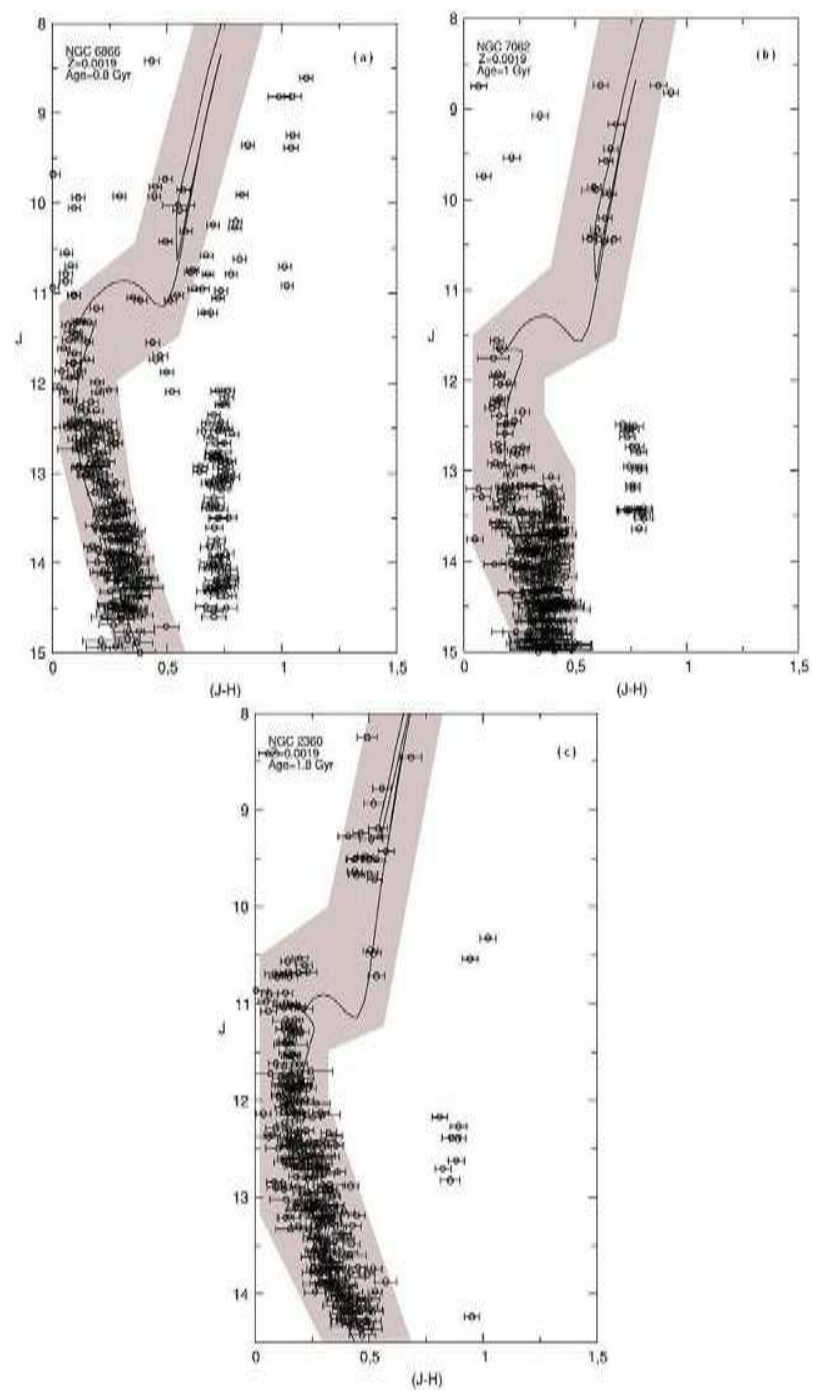

Figure 5: Observed decontaminated $J \times(J-H)$ CMDs extracted from the regions of $R=19^{\prime} .07$ for NGC $6866, R=7^{\prime} .53$ for NGC 7062 and $R=22^{\prime} .63$ for NGC 2360, respectively. The solid blue lines in the panels represent the fitted 0.8, 1, 1.8 Gyr Padova isochrones for $Z=+0.019$ (solar) abundance. The CMD filter used to isolate cluster MS/evolved stars is shown with the shaded area. 

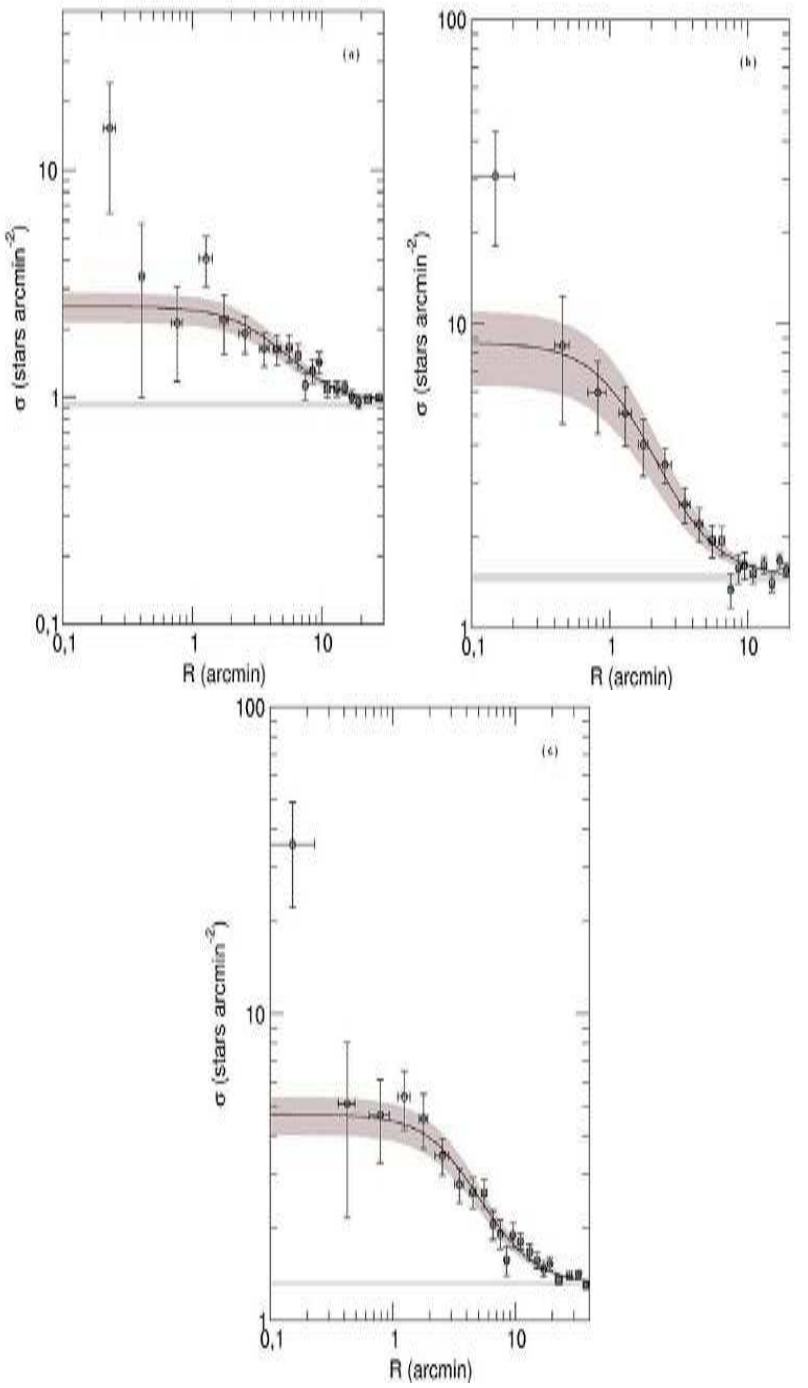

Figure 6: Stellar RDPs (open circles) of NGC 6866, NGC 7062, NGC 2360 built with CMD filtered photometry. Solid line shows the best-fit King profile. Horizontal red bar: stellar background level measured in the comparison field. Shaded region: $1 \sigma$ King fit uncertainty. 


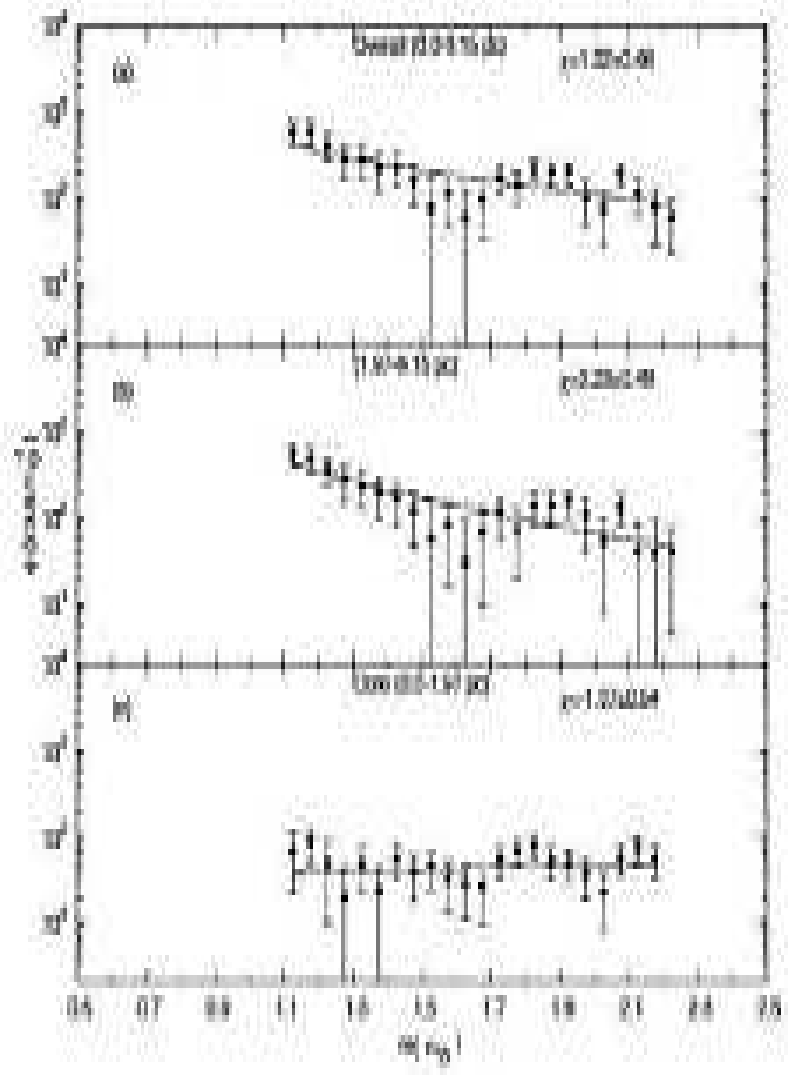

Figure 7: $\phi(m)\left(\right.$ stars $\left.m_{\odot}^{-1}\right)$ versus $m_{\odot}$ of NGC 6866 cluster, as a function of distance from the core. 


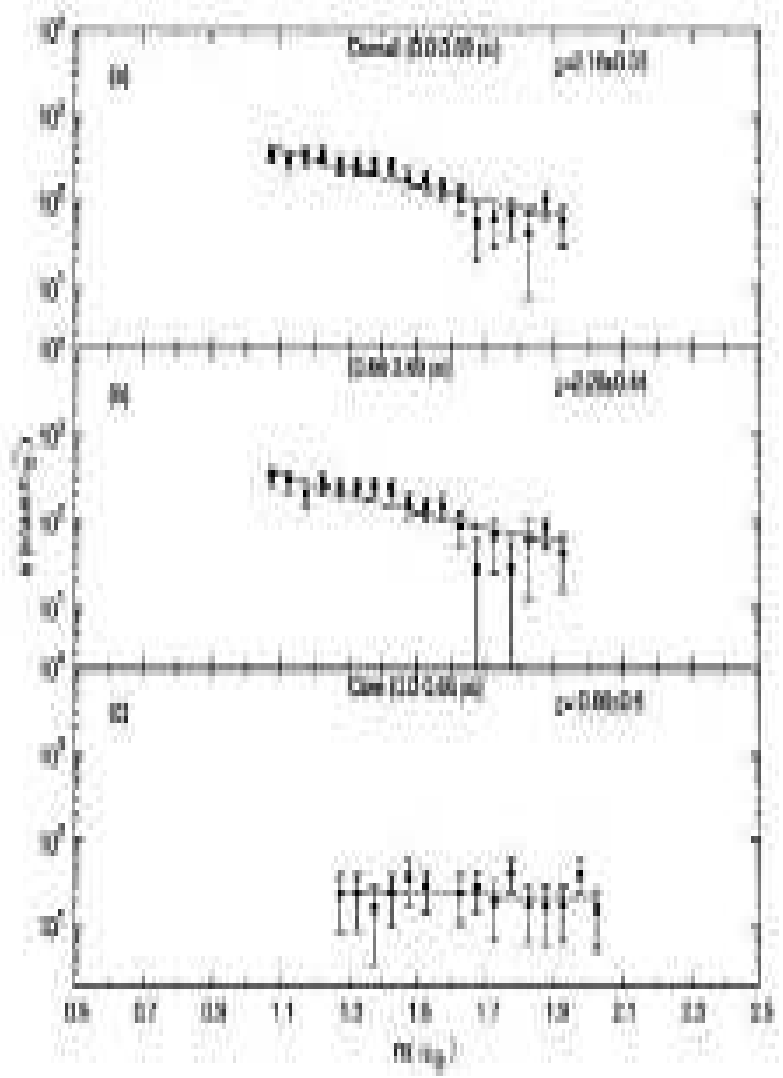

Figure 8: $\phi(m)\left(\right.$ stars $\left.m_{\odot}^{-1}\right)$ versus $m_{\odot}$ of NGC 7062 cluster, as a function of distance from the core. 


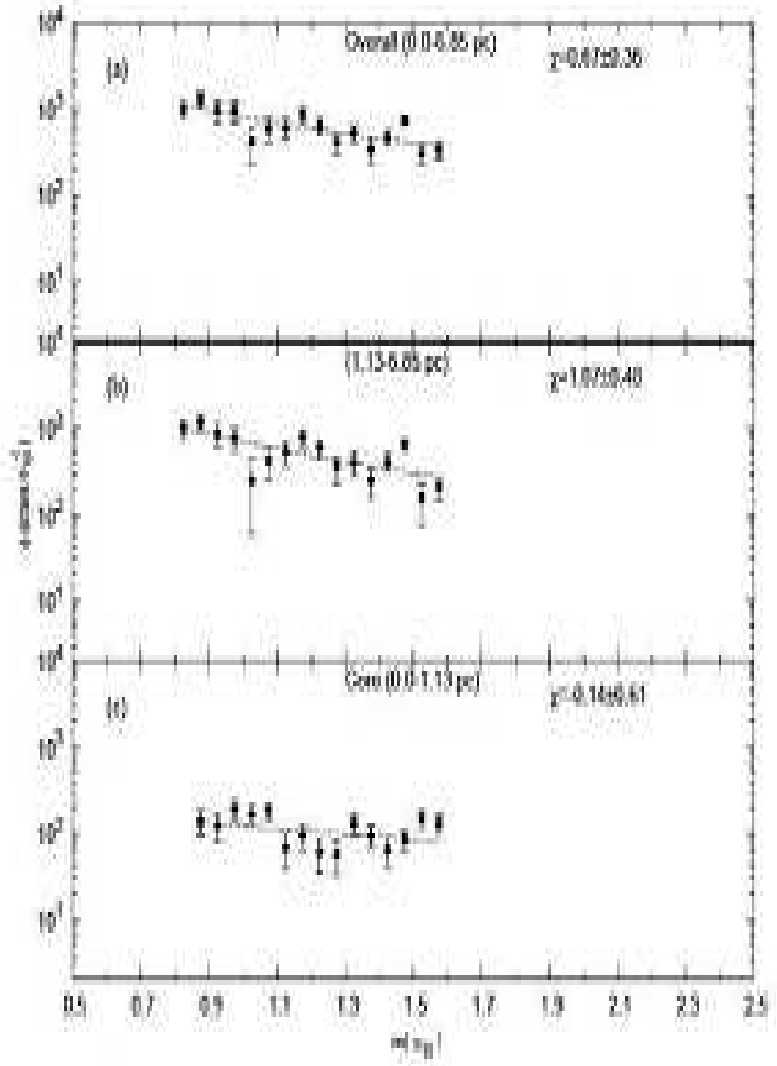

Figure 9: $\phi(m)\left(\right.$ stars $\left.m_{\odot}^{-1}\right)$ versus $m_{\odot}$ of NGC 2360 cluster, as a function of distance from the core. 


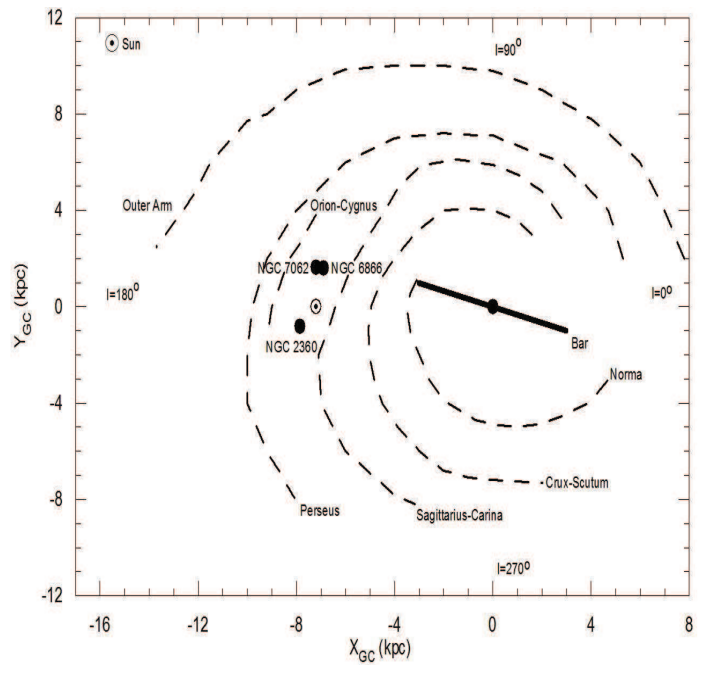

Figure 10: Spatial distribution of NGC 6866, NGC 7062, NGC 2360 clusters (filled circles). The schematic projection of the Galaxy is seen from the North pole. The Sun's distance to the Galactic center is taken to be $7.2 \mathrm{kpc}$ of Bica et al. (2006b). 


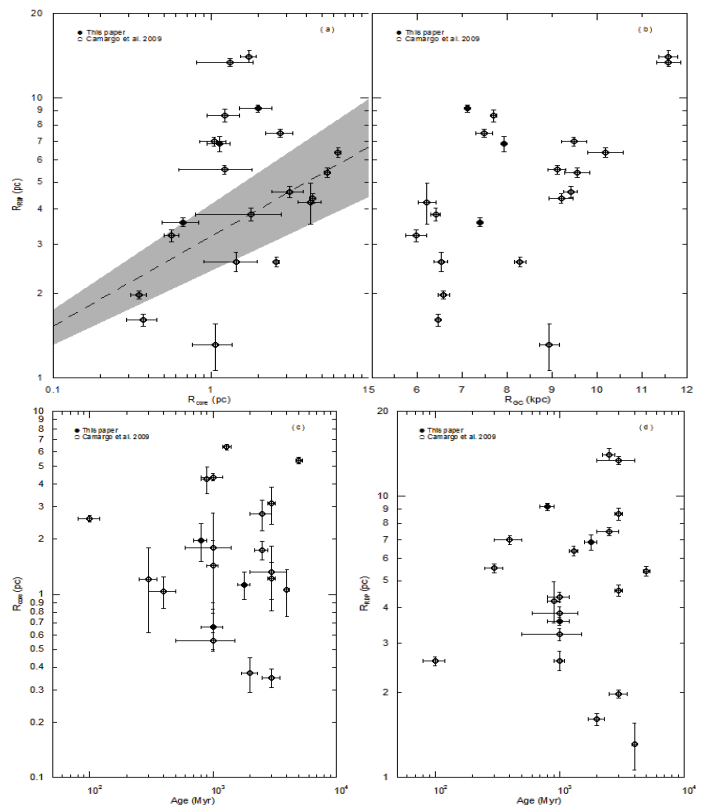

Figure 11: $R_{R D P}-R_{\text {core }}, R_{R D P}-R_{G C}, R_{\text {core }}-$ Age (Myr), and $R_{R D P}-$ Age (Myr) relations of NGC 6866, NGC 7062, NGC 2360 clusters. Filled and empty circles show the clusters of this work and Camargo et al. (2009), respectively. 
Table 1: Literature and presently optimised coordinates.

\begin{tabular}{|c|c|c|c|c|c|c|c|c|}
\hline \multirow[b]{2}{*}{ Cluster } & \multicolumn{4}{|c|}{ Literature } & \multicolumn{4}{|c|}{ This paper } \\
\hline & $\begin{array}{r}\alpha(2000) \\
(\mathrm{h} \mathrm{m} \mathrm{s})\end{array}$ & $\begin{array}{r}\delta(2000) \\
(0,1 \prime)\end{array}$ & $\begin{array}{l}\ell \\
\left({ }^{\circ}\right)^{\prime}\end{array}$ & $\begin{array}{l}b \\
\left({ }^{\circ}\right)\end{array}$ & $\begin{array}{r}(2000) \\
(\mathrm{h} \mathrm{m} \mathrm{s})\end{array}$ & $\begin{array}{r}\delta(2000) \\
(0,11)\end{array}$ & $\begin{array}{l}\ell \\
\left({ }^{\circ}\right)\end{array}$ & $\begin{array}{l}b \\
\left({ }^{\circ}\right)\end{array}$ \\
\hline NGC 6866 & 200355 & +440930 & 79.56 & 6.84 & $\begin{array}{llll}20 & 04 & 00.58\end{array}$ & +440913.50 & 79.56 & 6.82 \\
\hline NGC 7062 & $\begin{array}{lll}21 & 2327\end{array}$ & +462242 & 89.96 & -2.75 & $21 \quad 2322.64$ & +462348.76 & 89.96 & -2.73 \\
\hline NGC 2360 & $\begin{array}{lll}07 & 17 \quad 43\end{array}$ & $-1538 \quad 30$ & 229.81 & -1.43 & $\begin{array}{llll}07 & 17 & 42.95\end{array}$ & -153755.50 & 229.80 & -1.42 \\
\hline
\end{tabular}

Table 2: Derived fundamental astrophysical parameters, Age (Gyr), $E(B-V),(V-M v)_{o}, d$ (kpc) from 2 MASS JH $K_{s}$ photometry and the isochrones of $Z_{\odot} \cdot R_{G C}(\mathrm{kpc})$ in Col. 6 is the Galactocentric distance of three clusters.

\begin{tabular}{lccccc}
\hline Cluster & Age (Gyr) & $E(B-V)$ & $(V-M v)_{o}$ & $d(\mathrm{kpc})$ & $R_{G C}(\mathrm{kpc})$ \\
$(1)$ & $(2)$ & $(3)$ & $(4)$ & $(5)$ & $(6)$ \\
\hline NGC 6866 & $0.8 \pm 0.1$ & $0.19 \pm 0.06$ & $11.08 \pm 0.11$ & $1.65 \pm 0.09$ & $7.11 \pm 0.02$ \\
NGC 7062 & $1.0 \pm 0.2$ & $0.32 \pm 0.06$ & $11.07 \pm 0.11$ & $1.64 \pm 0.09$ & $7.40 \pm 0.02$ \\
NGC 2360 & $1.8 \pm 0.2$ & $0.06 \pm 0.03$ & $10.09 \pm 0.06$ & $1.04 \pm 0.03$ & $7.93 \pm 0.02$ \\
\hline
\end{tabular}

Table 3: Comparison of the fundamental astrophysical parameters of NGC 6866, NGC 7062, NGC 2360 to the literature. Cols. 1-4 represent the cluster name, reddening, the metal and heavy element abundances, respectively. True distance modulus values, $(V-M v)_{o}$, and their corresponding heliocentric distances are presented in Cols. 5-6, respectively. Cols. 7-8 give the age of $\log (A)$ and $\mathrm{A}(\mathrm{Gyr})$, respectively. The isochrones/observational ZAMSs and the photometry of the literature are listed in Cols. 9-10, respectively. The references of the literature are given in Col. 11.

\begin{tabular}{|c|c|c|c|c|c|c|c|c|c|c|}
\hline $\begin{array}{c}\text { Cluster } \\
(1)\end{array}$ & $\begin{array}{c}\mathrm{E}(\mathrm{B}-\mathrm{V}) \\
(2)\end{array}$ & $\begin{array}{c}{[\mathrm{Fe} / \mathrm{H}]} \\
(3)\end{array}$ & $\begin{array}{c}Z \\
(4) \\
\end{array}$ & $\begin{array}{c}(V-M v)_{O} \\
(5)\end{array}$ & $\begin{array}{c}d(\mathrm{kpc}) \\
(6)\end{array}$ & $\begin{array}{c}\log (A) \\
(7)\end{array}$ & $\begin{array}{c}A(G y r) \\
(8)\end{array}$ & $\begin{array}{c}\text { Iso/obs ZAMS } \\
(9)\end{array}$ & $\begin{array}{c}\text { Photometry } \\
(10)\end{array}$ & $\begin{array}{c}\text { Reference } \\
(11)\end{array}$ \\
\hline \multirow[t]{2}{*}{ NGC 6866} & 0.19 & - & 0.019 & 11.08 & 1.65 & 8.90 & 0.80 & G02 & 2 MASS & This paper \\
\hline & 0.17 & - & 0.019 & 10.80 & 1.45 & 8.58 & 0.38 & obs.ZAMS & UBV & [1] \\
\hline \multirow[t]{2}{*}{ NGC 7062} & 0.32 & - & 0.019 & 11.07 & 1.64 & 9.00 & 1.00 & G02 & 2 MASS & This paper \\
\hline & 0.46 & -0.35 & 0.003 & 12.18 & 2.73 & 8.45 & 0.28 & Vandenberg 1985 & $u v b y-\beta$ & {$[2]$} \\
\hline \multirow[t]{2}{*}{ NGC 2360} & 0.06 & - & 0.019 & 10.09 & 1.04 & 9.25 & 1.80 & G02 & 2 MASS & This paper \\
\hline & 0.07 & - & 0.019 & 10.30 & 1.15 & - & - & Eggen ZAMS & UBV & [3] \\
\hline
\end{tabular}

Table 4: Structural parameters of the clusters of NGC 6866, NGC 7062, NGC 2360.

\begin{tabular}{|c|c|c|c|c|c|c|c|c|c|c|c|}
\hline $\begin{array}{l}\text { Cluster } \\
\text { (1) }\end{array}$ & $\begin{array}{l}\left(1^{\prime}\right) \\
(p c) \\
(2)\end{array}$ & $\begin{array}{l}\sigma_{0 K} \\
\left(* p c^{-2}\right) \\
(3)\end{array}$ & $\begin{array}{l}\sigma_{b g} \\
\left(* p c^{-2}\right) \\
(4)\end{array}$ & $\begin{array}{l}R_{\text {core }} \\
(p c) \\
(5)\end{array}$ & $\begin{array}{l}R_{R D P} \\
(p c) \\
(6)\end{array}$ & $\begin{array}{l}\sigma_{0 K} \\
\left(*{ }^{\prime}-2\right) \\
(7)\end{array}$ & $\begin{array}{l}\sigma_{b g} \\
\left(*{ }^{\prime}-2\right) \\
(8)\end{array}$ & $\begin{array}{l}R_{\text {core }} \\
(\prime) \\
(9)\end{array}$ & $\begin{array}{l}R_{R D P} \\
(1) \\
(10)\end{array}$ & $\begin{array}{l}\Delta R \\
(1) \\
(11)\end{array}$ & $\mathrm{CC}$ \\
\hline NGC 6866 & 0.48 & $6.83 \pm 1.65$ & $4.08 \pm 0.12$ & $1.97 \pm 0.46$ & $9.15 \pm 0.27$ & $1.52 \pm 0.37$ & $0.94 \pm 0.02$ & $4.13 \pm 0.94$ & $19.07 \pm 0.57$ & $50-60$ & 0.90 \\
\hline NGC 7062 & 0.48 & $31.61 \pm 10.37$ & $6.45 \pm 0.20$ & $0.66 \pm 0.17$ & $3.59 \pm 0.13$ & $7.20 \pm 2.36$ & $1.47 \pm 0.05$ & $1.38 \pm 0.35$ & $7.53 \pm 0.27$ & $25-35$ & 0.89 \\
\hline NGC 2360 & 0.30 & $36.89 \pm 7.27$ & $14.45 \pm 0.26$ & $1.13 \pm 0.19$ & $6.85 \pm 0.44$ & $3.37 \pm 0.66$ & $1.32 \pm 0.02$ & $3.73 \pm 0.63$ & $22.63 \pm 1.44$ & $40-50$ & 0.93 \\
\hline
\end{tabular}


Table 5: The mass information for the clusters of NGC 6866, NGC 7062, NGC 2360.

\begin{tabular}{|c|c|c|c|c|c|c|c|}
\hline \multirow[b]{2}{*}{$\begin{array}{l}\text { Region } \\
\text { (pc) } \\
(1)\end{array}$} & \multicolumn{3}{|l|}{ Evolved } & \multicolumn{2}{|c|}{ Observed+Evolved } & \multicolumn{2}{|c|}{ Extrapolated+Evolved } \\
\hline & $\begin{array}{c}N^{*} \\
(\text { Stars }) \\
(2)\end{array}$ & $\begin{array}{c}m_{\text {evol }} \\
\left(10^{1} M_{\odot}\right) \\
(3)\end{array}$ & $\begin{array}{l}\chi \\
(4)\end{array}$ & $\begin{array}{c}N^{*} \\
\left(10^{2} \text { Stars }\right) \\
(5)\end{array}$ & $\begin{array}{c}m_{\text {obs }} \\
\left(10^{2} M_{\odot}\right) \\
(6)\end{array}$ & $\begin{array}{c}N^{*} \\
\left(10^{2} \text { Stars }\right) \\
(7)\end{array}$ & $\begin{array}{c}m_{t o t} \\
\left(10^{2} M_{\odot}\right) \\
(8)\end{array}$ \\
\hline & NGC 6866 & & $m=1.13-2.23$ & & & & \\
\hline $0.00-1.97$ & $6 \pm 3$ & $1.3 \pm 0.6$ & $-1.37 \pm 0.54$ & $0.65 \pm 0.07$ & $1.12 \pm 0.40$ & $1.04 \pm 0.50$ & $1.38 \pm 0.44$ \\
\hline $1.97-9.15$ & $7 \pm 8$ & $1.5 \pm 1.7$ & $2.23 \pm 0.49$ & $2.01 \pm 0.24$ & $3.02 \pm 0.87$ & $48.8 \pm 37.5$ & $16.9 \pm 7.15$ \\
\hline $0.00-9.15$ & $13 \pm 8$ & $2.8 \pm 1.9$ & $1.32 \pm 0.46$ & $2.59 \pm 0.25$ & $4.05 \pm 1.13$ & $48.8 \pm 37.0$ & $17.8 \pm 7.08$ \\
\hline & NGC 7062 & & $m=1.08-1.93$ & & & & \\
\hline $0.00-0.66$ & $1 \pm 1$ & $0.1 \pm 0.2$ & $-0.66 \pm 0.60$ & $0.33 \pm 0.04$ & $0.53 \pm 0.23$ & $1.08 \pm 2.49$ & $0.98 \pm 0.72$ \\
\hline $0.66-3.59$ & $10 \pm 5$ & $2.1 \pm 1$ & $2.28 \pm 0.44$ & $1.65 \pm 0.12$ & $2.33 \pm 0.45$ & $39.7 \pm 30.2$ & $13.5 \pm 5.66$ \\
\hline $0.00-3.59$ & $11 \pm 5$ & $2.2 \pm 1.1$ & $2.16 \pm 0.33$ & $1.94 \pm 0.13$ & $2.73 \pm 0.42$ & $45.7 \pm 34.5$ & $15.6 \pm 6.43$ \\
\hline & NGC 2360 & & $m=0.83-1.63$ & & & & \\
\hline $0.00-1.13$ & $22 \pm 5$ & $3.5 \pm 0.8$ & $-0.14 \pm 0.61$ & $1.15 \pm 0.09$ & $1.46 \pm 0.21$ & $=$ & $=$ \\
\hline $1.13-6.85$ & $19 \pm 12$ & $3.0 \pm 1.9$ & $1.07 \pm 0.48$ & $4.74 \pm 0.37$ & $5.38 \pm 0.58$ & $51.9 \pm 39.8$ & $18.1 \pm 7.49$ \\
\hline $0.00-6.85$ & $41 \pm 13$ & $6.5 \pm 2.1$ & $0.67 \pm 0.36$ & $5.81 \pm 0.37$ & $6.77 \pm 0.60$ & $47.1 \pm 33.5$ & $18.1 \pm 6.24$ \\
\hline
\end{tabular}

and 8 , respectively.

Table 6: Relaxation time and evolutionary parameter

\begin{tabular}{ccccc}
\hline \hline \multirow{2}{*}{ Cluster } & \multicolumn{2}{c}{ Core } & \multicolumn{3}{c}{ Overall } \\
\cline { 2 - 5 }$(1)$ & $\begin{array}{c}t_{\text {relax }} \\
(\text { Myr })\end{array}$ & $\tau$ & \multicolumn{2}{c}{$\begin{array}{c}t_{\text {relax }} \\
\text { Myr })\end{array}$} \\
(2) & $(2)$ & $(3)$ & $(4)$ & $(5)$ \\
\hline NGC 6866 & $1.77 \pm 0.78$ & $453 \pm 207$ & $210 \pm 141$ & $3.80 \pm 2.59$ \\
NGC 7062 & $0.61 \pm 1.12$ & $1640 \pm 3020$ & $77.8 \pm 52.0$ & $12.8 \pm 8.96$ \\
NGC 2360 & $3.17 \pm 71.7$ & $569 \pm 12900$ & $153 \pm 96.1$ & $11.8 \pm 7.55$ \\
\hline
\end{tabular}




\section{Acknowledgments}

We thank the anonymous referee for her/his comments and suggestions. This publication makes use of data products from the Two Micron All Sky Survey, which is a joint project of the University of Massachusetts and the Infrared Processing and Analysis Centre/California Institute of Technology, funded by the National Aeronautics and Space Administration and the National Science Foundation. This research has made use of the WEBDA database, operated at the Institute for Astronomy of the University of Vienna. This work was supported by the Research Fund of the University of Istanbul, Project number: BYP-22411.

\section{References}

Bica, E., Bonatto Ch., Blumberg, R., 2006a, A\&A, 460, 83

Bica, E., Bonatto, Ch., Barbuy, B., Ortolani, S., 2006b, A\&A, 450, 105

Bica, E., Bonatto, Ch., Camargo, D., 2008, MNRAS, 385, 349

Bica, E., Bonatto, Ch., Dutra, C., 2008, A\&A, 489, 1129

Binney, J. \& Merrifield, M. 1998, in Galactic Astronomy, Princeton, NJ: Princeton University Press. (Princeton series in astrophysics)

Bonatto, Ch., Bica, E., Girardi, L., 2004, A\&A, 415, 571

Bonatto, Ch., Bica, E., 2005, A\&A, 437, 483

Bonatto, Ch., Bica, E., 2006, A\&A, 455, 931

Bonatto, Ch., Bica, E., 2007a, A\&A, 473, 445

Bonatto, Ch., Bica, E., 2007b, MNRAS, 377, 1301

Bonatto, C., Bica, E, 2008, A\&A, 485, 81

Bonatto, Ch., Bica, E., 2009a, MNRAS, 392, 483

Bonatto, Ch., Bica, E., 2009b, MNRAS, 394, 2127

Bonatto, Ch., Bica, E., 2009c, MNRAS, 397, 1915

Bonifacio, P., Monai, S., Beers, T.C., 2000, AJ, 120, 2065

Camargo, D., Bonatto, C., Bica, E., 2009, A\&A, 508, 211

Camargo, D., Bonatto, C., Bica, E, 2010, A\&A, 521, 42

Dutra, C.M., Santiago, B.X., Bica, E., 2002, A\&A, 383, 219

Elson, R.A.W., Fall, S.M., \& Freeman, K.C., 1987, ApJ, 323, 54 
Eggen, O., 1968, ApJ, 152, 83

Gieles, M., Portegies-Zwart, S., Athanassoula, E., Baumardt, H., Lamers, H.J.G.L.M., Sipior, M, Leenaarts, J., 2006, MNRAS, 371, 793

Gieles, M., Athanassoula, E, Portegies-Zwart, S. 2007, MNRAS, 376, 809

Hoag, A.A., Johnson, H.L., Iriarte, B., Mitchell, R.I., Hallam, K.L., Sharpless, S. Publications of the U.S. Naval Observatory. 2d ser., v.17, pt. 7, Washington, U.S. Govt. Print. Off., 1961, p.344-542

King, I., 1962, AJ, 67, 471

King, I., 1966, AJ, 71, 64

Kroupa, P. 2001, MNRAS, 322, 231

Lamers, H.J.G.L.M., Gieles, M., 2006, A\&A, 455, 17

Mackey, A.D., Wilkinson, M.I., Davies, M.B., Gilmore, G.F., 2007, MNRAS, 379,40

Peniche, R., Pena, J.H., Diaz Martinez, S.H., Gomez, T., 1990, RMxAA, 20, 127

Salpeter, E. 1955, ApJ, 121, 161

Schlegel, D.J., Finkbeiner, D. P., \& Davis, M., 1998, ApJ, 500, 525

Skrutskie, M.F., Cutri, R., Stiening, R., Weinberg, M.D., Schneider, S.E., Carpenter, J.M., Beichman, C., Capps, R., 2006, AJ, 131, 1163

Wilson, C.P., 1975, AJ, 80, 175

Vandenberg, D. A., Bell, R. A., 1985, ApJS, 58, 561

van den Berg, S., Morbey, C., Pazder, J., 1991, ApJ, 375, 594 